\title{
Neuroimaging reveals functionally distinct neuronal networks associated with high level alcohol consumption in two genetic rat models
}

Short title: Strain-specific functional brain activation by alcohol in rats

Vicente Pallarés ${ }^{1, \ddagger}$, Mateusz Dudek ${ }^{2, \ddagger}$, Andrea Moreno $^{1}$, Úrsula Pérez-Ramírez ${ }^{3}$, David Moratal ${ }^{3}$, Mia Haaranen ${ }^{2}$, Roberto Ciccocioppo ${ }^{4}$, Wolfgang H Sommer ${ }^{5}$, Santiago Canals ${ }^{1, *}$, Petri Hyytiä ${ }^{2, *}$

${ }^{1}$ Instituto de Neurociencias, Consejo Superior de Investigaciones Científicas \& Universidad Miguel Hernández, Sant Joan d'Alacant, Spain

${ }^{2}$ Department of Pharmacology, Medicum, University of Helsinki, Helsinki, Finland

${ }^{3}$ Center for Biomaterials and Tissue Engineering, Universitat Politècnica de València, Valencia, Spain

${ }^{4}$ School of Pharmacy, Pharmacology Unit, University of Camerino, Camerino, Italy

${ }^{5}$ Institute of Psychopharmacology, Central Institute of Mental Health, University of Heidelberg, Mannheim, Germany

¥Contributed equally to this work.

*Contributed equally to this work.

Corresponding authors: Petri Hyytiä (petri.hyytia@helsinki.fi), Santiago Canals (scanals@umh.es)

Petri Hyytiä

Department of Pharmacology

POB 63

00014 University of Helsinki

Finland

Email: petri.hyytia@helsinki.fi

The authors declare no competing financial interests. The work was supported under the ERA-Net NEURON framework with grants given to the project "Translational Neuroimaging in Alcoholism" (TRANSALC) by the Academy of Finland (TRANSALC 01EW1112), and the Spanish Ministry of Science and Innovation (PIM2010ERN-00679). Further funding was obtained from the Spanish State Research Agency through the Severo Ochoa Program for Centres of Excellence in R\&D (SEV- 20170723), the Ministerio de Economía y Competitividad (MINECO) and FEDER funds (BFU2015-64380C2-1-R, BFU2015-64380-C2-2-R), the Generalitat Valencia through the Prometeo Program (PROMETEO/2019/015), the Spanish Ministerio de Sanidad, Servicios Sociales e Igualdad (\#20171065), the Bundesministerium für Bildung und Forschung (FKZ01EW1112), the Deutsche Forschungsgemeinschaft (DFG, German Research Foundation) - Project-ID 402170461 - TRR 265 B02 (Heinz et al., 2020), and the European Union's Horizon 2020 program (668863-SyBil-AA). 


\section{ABSTRACT}

Human imaging data suggest that the motivational processes associated with alcohol reward are reflected in the patterns of neural activation after alcohol or alcohol-related cues. In animal models of alcohol drinking, however, the changes in brain activation during voluntary alcohol ingestion are poorly known. In order to improve the translational utility of animal models, we examined alcoholinduced functional brain activation in AA (Alko Alcohol) and Marchigian-Sardinian alcohol-preferring (msP) rats that drink voluntarily high levels of alcohol, but exhibit widely different neurochemical and behavioral traits co-segregated with alcohol preference. Brain imaging was performed using manganese-enhanced magnetic resonance imaging (MEMRI), which is based on accumulation of $\mathrm{Mn}^{2+}$ ions in activated neurons, allowing the identification of functional neuronal networks recruited during specific behaviors in awake animals during a subsequent imaging session under anesthesia. MEMRI was performed following four weeks of voluntary alcohol drinking, using water drinking as the control. Despite similar levels of alcohol drinking, strikingly different alcohol-induced neuronal activity patterns were observed in AA and msP rats. Overall, functional activation in the AA rats was more widespread, involving large cortical areas and subcortical structures, such as the bed nucleus of the stria terminalis, preoptic area, hypothalamus, periaqueductal grey, and substantia nigra. In the msP rats, however, alcohol-related activation was largely confined to prefrontal cortical regions and insular cortex, and olfactory areas. Overlapping areas of activation found in both rat lines included the nucleus accumbens, prelimbic, orbital, and insular cortex. In conclusion, our data reveal strikingly different brain circuits associated with alcohol drinking in two genetically different rat lines and suggest innately different motivational and behavioral processes driving alcohol drinking. These findings have important implications for the use of these lines in translational alcohol research.

KEYWORDS: alcohol drinking; functional brain activity; selected rat lines; manganese-enhanced magnetic resonance imaging

INTRODUCTION 
Functional brain imaging in humans reveals that patients suffering from alcohol use disorders (AUD) display differences from healthy controls in brain systems associated with reward and motivation, as well executive and inhibitory control. In AUD patients, alcohol or alcohol-associated cues activate ventral or dorsal striatum, regions of the prefrontal cortex, insula, and thalamus (Myrick et al., 2004; Filbey et al., 2008; Gilman et al., 2008; Vollstadt-Klein et al., 2010; Gilman et al., 2012a; Gilman et al., 2012b; Courtney et al., 2016; Bach et al., 2020). Functional neuroimaging has also begun to identify biomarkers for AUD pharmacotherapies by evaluating brain activation changes induced by pharmacological treatments (Grodin and Ray, 2019). These studies have both highlighted the mechanisms of action of the pharmacological agents used for treatment and predicted clinical response (Schacht et al., 2017; Bach et al., 2020).

The findings in AUD are compatible with the neurocircuitry of alcohol reinforcement and dependence established with various methods in rodents (Spanagel, 2009). Rodents will voluntarily drink alcohol to attain pharmacologically and behaviorally relevant blood alcohol concentrations, and the ensuing activation of brain circuits can be measured. However, brain activation measured noninvasively in freely moving subjects would greatly enhance the translational use of data from animal models. Neuroimaging can objectively measure activity in brain circuits involved in alcohol drinking and both elucidate the neural circuitry underlying the incentives to drink alcohol and offer biomarkers of the actions of novel pharmacological agents for further human testing. Thus, biomarkers from in vivo neuroimaging methods can provide objective measures of distinct animal phenotypes that allow more straightforward translation into humans than behavioral responses, and may thus provide highly needed translational biomarkers (Heilig et al., 2016).

The use of in vivo neuroimaging techniques in rodents for mapping brain activity, including Magnetic Resonance Imaging (MRI), has been limited by the necessity of anesthesia for animal fixation in imaging settings. However, the emerging manganese-enhanced MRI (MEMRI) protocols offer a feasible approach (Silva et al., 2004). Paramagnetic $\mathrm{Mn}^{2+}$ ions enter excitable cells during depolarization through voltage-gated calcium channels. The activity-dependent accumulation of 
$\mathrm{Mn}^{2+}$ in neurons reports activated brain areas as increased signal intensity in $\mathrm{T}_{1}$-weighted $\mathrm{MR}$ images. Because $\mathrm{Mn}^{2+}$ does not leave the areas for several hours, the pattern of activation is retained. Therefore, neuronal activation resulting from behavioral activity in freely moving animals can be subsequently measured under anesthesia using MEMRI (Eschenko et al., 2010b; Dudek et al., 2015; Dudek et al., 2016).

Selective breeding was an early approach for developing rodent models consuming high amounts of alcohol (Mardones et al., 1953; Eriksson, 1968). Many breeding programs produced rat lines that quickly learn to drink voluntarily high amounts of alcohol $(5-8 \mathrm{~g} / \mathrm{kg} /$ day $)$ that can lead to blood alcohol levels as high as $100 \mathrm{mg} \%(0.1 \mathrm{~g} / 100 \mathrm{ml})$ (Ciccocioppo, 2013). Despite their similar alcohol preference phenotypes, rats from different selection programs differ in distinct neurochemical and behavioral traits which have co-segregated with their alcohol preference. For example, the Finnish alcohol-preferring AA rats are impulsive and risk-taking (Moller et al., 1997; Roman et al., 2007), whereas the Sardinian preferring line and its substrain, the Marchigian-Sardinian alcohol-preferring rats (msP), exhibit an anxiogenic- and depression-like phenotype (Colombo et al., 1995; Hansson et al., 2006). These rats display a constitutive overexpression of brain CRF1 receptors that is normalized following voluntary alcohol drinking (Hansson et al., 2006; Hansson et al., 2007).

Here, we hypothesized that MEMRI could capture neuronal activity patterns resulting from voluntary alcohol drinking by the alcohol-preferring rat lines. We then aimed at comparing functional brain activation in the $\mathrm{AA}$ and $\mathrm{msP}$ rats in response to alcohol consumption. Given their widely different behavioral traits besides similar alcohol-drinking phenotypes, differences in brain activation may help to decipher disparate motivational mechanisms involved in their alcohol consumption. Because alcoholism is an etiologically and clinically heterogeneous disorder (Heilig et al., 2011), comparison of the genetically different high-alcohol drinking phenotypes could highlight neurobiological mechanisms underlying this diversity and present brain activation biomarkers for testing the therapeutic potential of novel medications. 


\section{METHODS}

\section{Animals}

Twenty male alcohol-preferring AA (Alko, Alcohol) rats (265-335 g at the beginning of the experiments) and $28 \mathrm{msP}$ rats (370-480 g) were used. Experiments with AA rats were performed at the Department of Pharmacology of the University of Helsinki, and msP rat experiments were performed at the Neuroscience Institute of the Superior Research Council of Spain and Miguel Hernández University. In all cases, rats were individually housed in transparent polycarbonate cages with bedding material, and a wooden stick and nesting material were given as enrichment. Rats had ad libitum access to food and water and were housed under controlled temperature $\left(21 \pm 1^{\circ} \mathrm{C}\right)$ and relative humidity $(55 \pm 10 \%)$ on a 12 -hour light/dark cycle. All experimental procedures using animals were approved by local authorities (State Provincial Office of Southern Finland and IN-CSIC ethical committee, respectively) and carried out in accordance with the European Union Commission recommendation (2007/526/EC), the Finnish Act on the Use of Animals for Experimental Purposes and the Spanish law 32/2007.

\section{Voluntary alcohol consumption}

Drinking fluids were provided in 250-ml drinking bottles equipped with stainless steel spouts, placed in the food tray of the cage covers. During the first 4 days, AA rats of the alcohol group were given $10 \%(\mathrm{v} / \mathrm{v})$ ethyl alcohol as their only drinking fluid to habituate them to the taste of alcohol. This habituation is a standard procedure in the AA rats, but has not deemed necessary for msP rats. All rats were allowed a 2-bottle choice between $10 \%(\mathrm{v} / \mathrm{v})$ alcohol and water for the next 4 weeks, during which alcohol consumption reached a plateau. Twice per week, consumption of the fluids and body weights were recorded, and the bottles were filled with fresh solutions. During this phase, 
the water-drinking control group had always access to water in two drinking bottles. During the $\mathrm{MnCl}_{2}$ infusion, liquid consumption and body weights were recorded daily.

\section{$\mathrm{MnCl}_{2}$ administration}

Different doses and administration protocols for $\mathrm{MnCl}_{2}$ (Eschenko et al., 2010b; Eschenko et al., 2010a) were first tested in both rat strains in order to optimize MEMRI experiments in combination with alcohol drinking paradigms. The optimatization process traded off between two factors: achievement of minimal or no $\mathrm{MnCl}_{2}$ side effects, measured in general behavioral parameters with special attention to the achieved drinking levels, and maximization of $\mathrm{T}_{1}$-weighted signal enhancement in $\mathrm{MR}$ images. In all cases $\mathrm{MnCl}_{2}$ was administered as an isotonic solution dissolved in Tris-buffered saline ( $\mathrm{pH}$ 7.4). As a consequence of this optimization process, we chose osmotic minipump administration (ALZET ${ }^{\circledR}$ model 2001) delivering $200 \mu \mathrm{l}$ of $\mathrm{MnCl}_{2}(1 \mu \mathrm{l} / \mathrm{h})$ during a 7-day infusion period. The total dose of infused $\mathrm{MnCl}_{2}$ was $120 \mathrm{mg} / \mathrm{kg}$ for AA and $80 \mathrm{mg} / \mathrm{Kg}$ for $\mathrm{msP}$ rats, increasing the signal-to-noise ratios (SNR) in imaging data by a factor of 1.25 and 1.40 , respectively, with no toxic effects (see Results section). Before the surgery, the pumps were primed overnight in a $37^{\circ} \mathrm{C}$ saline solution. The pumps were implanted subcutaneously on the dorsum, slightly caudal to the scapulae. The animals received post-surgical analgesia (Carprofen, $5 \mathrm{mg} / \mathrm{kg}$ ) immediately after implantation and were returned to their home cages with access to water only (control groups) or both alcohol and water bottles (alcohol-exposed groups). All animals were imaged immediately following the 7-day $\mathrm{MnCl}_{2}$ infusion within $24 \mathrm{~h}$ of termination of alcohol access.

\section{MRI data acquisition}

Anesthesia was induced with $4-5 \%$ isoflurane in oxygen $(0.8-1 \mathrm{~L} / \mathrm{min})$, and animals were secured on custom-made holding apparatus with a tooth bar and a nose cone. During scanning, the isoflurane 
concentration was maintained at $2 \%$, and the body temperature was kept constant with a heating pad.

MRI experiments on AA rats were performed on a $4.7 \mathrm{~T}$ scanner (Bruker, PharmaScan 47/16, Ettlingen, Germany) using a $38 \mathrm{~mm}$ linear volume coil for transmit and receive. $\mathrm{T}_{1}$-weighted images were acquired using a rapid acquisition-relaxation enhanced (RARE) pulse sequence (TR $=300 \mathrm{~ms}$, $\mathrm{TE}=12.5 \mathrm{~ms}$, averages $=7$, field of view FOV $=26 \times 17 \times 17 \mathrm{~mm}$, matrix size $=128 \times 54 \times 54$, resulting in $0.2 \times 0.31 \times 0.31 \mathrm{~mm}$ voxel resolution).

MRI experiments on msP rats were performed on a $7 \mathrm{~T}$ scanner (Bruker, BioSpect 70/30, Ettlingen, Germany) using a receive-only phase array coil with integrated combiner and preamplifier in combination with an actively detuned transmit-only resonator. $T_{1}$-weighted images were acquired using a multi-slice multi-echo (MSME) pulse sequence $(T R=300 \mathrm{~ms}, \mathrm{TE}=14 \mathrm{~ms}$, averages $=8$, field of view FOV $=32 \times 32$ and 9 horizontal slices of $0.5 \mathrm{~mm}$ thickness, matrix size $=256 \times 256$, resulting in $0.125 \times 0.125 \times 0.5 \mathrm{~mm}$ voxel resolution).

\section{Data analysis}

All MRI datasets were analyzed at the Neuroscience Institute (CSCI-UMH) following the same pipeline. The MRI images were converted to Analyze 7.5 format and scaled up by a factor of 10 . All images were preprocessed with custom-developed MATLAB functions (version R2011a). In brief, brain-extracted (FSL BET, Oxford Centre for Functional Magnetic Resonance imaging of the Brain, London, UK) (Smith, 2002) $\mathrm{T}_{1}$-weighted images were co-registered and further registered to a stereotaxic rat brain MRI template (Schwarz et al., 2006) by a 12-parameter affine transformation using the FSL/FLIRT tool (Jenkinson et al., 2002). The template is co-registered to a digitized atlas (Paxinos and Watson, 2007), which enabled atlas-based generation of region-of-interest (ROI) masks for detailed anatomical analysis. The resulting images were smoothed to improve signal-to-noise 
ratio using a Gaussian kernel with $4 \times 4 \times 4 \mathrm{~mm}$ FWHM for both msP and AA. For identifying the brain regions in which activation in the alcohol-drinking groups differed from the water-drinking control group, voxel-wise independent t-tests were performed in SPM8 (www.fil.ion.ucl.ac.uk/spm/). For pump infusion experiments in AA and msP rats, the arbitrary significance threshold was set to $P<0.01$ (uncorrected) for individual voxels, and then a cluster size threshold was applied to correct for multiple comparisons at $P<0.05$. The cluster size threshold value was determined using Monte Carlo simulation ( 54 and 13 voxels for AA and msP rats, respectively). For the acute i.p $\mathrm{MnCl}_{2}$ administration experiment in msP rats, a significance level of $\mathrm{P}<0.05$ corrected for multiple comparison was defined (cluster size of 86 voxels defined by Monte Carlo simulation). The same data were also tested for lower or higher $p$-values. The thresholded statistical maps were overlaid on a common anatomical template.

\section{STATISTICS}

To test the possible toxic effects of $\mathrm{MnCl}_{2}$ infusion on alcohol drinking, daily alcohol intake was testing using one-way repeated measures analysis of variance (ANOVA) in both rat strains. In addition, the strains were compared using a two-way (strain, time) ANOVA, with repeated measures on time. In the repeated measures ANOVAs, assumption of sphericity was tested with Mauchly's sphericity test. If sphericity was violated, the degrees of freedom of the F-distribution were corrected with the Greenhouse-Geisser procedure. The level for statistical significance was set at $p$ $<0.05$.

RESULTS

Behavioral effects of $\mathrm{MnCl}_{2}$ infusion 
At the baseline before implantation of $\mathrm{MnCl}_{2}$-contaíning osmotic minipumps, $\mathrm{AA}$ and msPrat strains reached similar levels of daily alcohol consumption, approximately $4-5 \mathrm{~g} / \mathrm{kg}$ body weight/day. Manganese administration produced no observable behavioral alteration, including locomotor activity. One-way ANOVA indicated that either the $\mathrm{MnCl}_{2}$ infusion, the surgical procedures, or both altered alcohol drinking in the AA rats $\left(F_{2.33,21.0}=4.19, p=0.025\right)$. The reduction in alcohol intake was transient, reaching statistical significance during the second and third infusion days. By the fourth day, the intake returned to the baseline (Fig. 1). No significant changes in alcohol drinking were found in the msP rats $\left(F_{2.45,31.84}=0.37, p=0.74\right)$ and the comparison of AA and msP rats revealed no differences between them $\left(F_{1,22}=0.53, p=0.47\right)$.

Functional mapping of brain activity during alcohol drinking in AA rats

Statistical parametric maps presented in Fig. 2 show statistically significant $(P<0.05$, corrected) increase of $\mathrm{T}_{1}$-signal intensity in AA alcohol drinking rats compared to the water-drinking control animals. No decreases in $T_{1}$-signal were found statistically significant. Enhanced $T_{1}$ intensity reflects accumulation of $\mathrm{Mn}^{2+}$ into brain tissue and therefore suggests increased functional brain activation. In the forebrain, alcohol-induced activation was observed bilaterally in the nucleus accumbens, anterior insula, regions of the piriform cortex, and medially in the anterior cingulate cortex. The striatal activation cluster belonged to a continuum that extended to the ventral pallidum, preoptic area, bed nucleus of the stria terminalis, anterior hypothalamus, and the perifornical area of lateral hypothalamus. Both dorsal and ventral regions of hippocampus displayed activation. In the midbrain, activation was found in the substantia nigra and periaqueductal grey. The largest percentwise activation was seen in the ecto- and perirhinal cortices, as well as the temporal association cortex, that consisted of a unilateral cortical activation cluster (Fig. 4). Other cortical areas displaying activation included the primary and secondary motor and somatosensory cortices. 


\section{Functional mapping of brain activity during alcohol drinking in $\mathrm{ms} P$ rats}

Statistical parametric maps presented in Fig. 3 show statistically significant $(P<0.05$, corrected) increase of $\mathrm{T}_{1}$-signal intensity in $\mathrm{msP}$ alcohol drinking rats compared to the water-drinking control group. As for AA rats, no decreases in $\mathrm{T}_{1}$-signal were found. Activity increases revealed by osmotic mini-pump infusion of $\mathrm{MnCl}_{2}$ were largely confined to prefrontal cortical regions including the cingulate, prelimbic, infralimbic, peduncular and orbitofrontal cortex, as well as the insular cortex. Several olfactory areas including the olfactory bulb, accessory olfactory bulb, anterior olfactory nucleus, tenia tecta, and piriform cortex were identified. Activity increases in subcortical regions were mainly restricted to the nucleus accumbens.

\section{DISCUSSION}

Our present data demonstrate the utility of MEMRI for capturing brain activation patterns accumulated over several days in freely behaving animals, in agreement with previous studies using this imaging technique. Most importantly, we showed remarkable differences in brain activation between two rat lines, $A A$ and $\mathrm{msP}$ rats, selected for high alcohol drinking and displaying identical alcohol drinking levels in the current experiments. The level of voluntary alcohol drinking by these rat lines has previously been shown to induce psychomotor stimulatory, antidepressant, and anxiolytic actions (Paivarinta and Korpi, 1993; Colombo et al., 1998; Ciccocioppo et al., 2006). Furthermore, prolonged alcohol consumption lead to persistent brain activation changes during early abstinence (Dudek et al., 2015), and to white and grey matter integrity alterations that paralleled those found in human alcoholics (De Santis et al., 2019; De Santis et al., 2020). Together, these behavioral and neural consequences of high and prolonged alcohol drinking in these lines model several important features of the trajectory of human AUD and suggest that the brain imaging data obtained from these models could have translational value. 
Differential $\mathrm{Mn}^{2+}$ accumulation between the alcohol-exposed and control groups can most likely be attributed both to the direct neurochemical effects of ingested alcohol and the activity of brain circuits underlying the incentives to drink alcohol. As previously described, administration of $\mathrm{MnCl}_{2}$ with continuous systemic infusion produced no toxicity and only minor behavioral alterations (Eschenko et al., 2010b; Eschenko et al., 2010a; Dudek et al., 2015). Together, these data suggest that MEMRI is a versatile approach for studying various alcohol-related behaviors, and the ease of obtaining enhanced T1 contrasts allows comparison of data across different imaging platforms. Additional benefits of MEMRI for mapping brain activity include its independency from the nonlinearities introduced by neurovascular coupling in imaging techniques such as fMRI with BOLD contrast, and the high spatial resolution provided by the robust MR sequences used for $\mathrm{T}_{1}$-weighted imaging. The use of anesthesia during MEMRI can be considered a confounding factor. On the other hand, isoflurane reduces neuronal excitability and it is not likely that isoflurane during a 1-hour imaging session could significantly alter the neuronal $\mathrm{Mn}^{2+}$ concentrations accumulated over several days. When $\mathrm{Mn}^{2+}$ is administered as continuous infusion as in the present experiments, the temporal resolution of the method is poor. However, our aim was not to map acute effects of alcohol ingestion, but to characterize the networks repeatedly activated during long-term alcohol drinking. MEMRI has been increasingly exploited for identifying neural circuits associated with specific behaviors in similar experimental settings (Eschenko et al., 2010b; Gildish et al., 2012; Bangasser et al., 2013; McGuire et al., 2013; Laine et al., 2017; Yang and Li, 2020).

Although our most striking finding was the engagement of widely different circuits in AA and msP rats, we also saw important overlaps in regional brain activity. Activation of common neural substrates by alcohol in these lines was expected because similar neurochemical actions of alcohol have been found across various genetic models of alcohol-drinking (McBride and Li, 1998; Ciccocioppo et al., 2006; Sommer et al., 2006). One of these structures was the nucleus accumbens that has long been implicated in the reinforcing effects of drugs of abuse, including alcohol (Koob et al., 1998). The nucleus accumbens activation revealed by MEMRI is also in line with previous 
attempts to map global brain activation by alcohol using either deoxyglucose metabolic mapping (Porrino et al., 1998) or c-Fos immunohistochemistry (Bachtell et al., 1999; Hansson et al., 2008; Vilpoux et al., 2009). Furthermore, either experimenter-administered or self-administered alcohol increased extracellular dopamine release in the nucleus accumbens both in the AA and SP rats, the parental strain of msPs (Nurmi et al., 1998; De Montis et al., 2004). The ventral striatal activation has been consistently associated with presentation of alcohol or alcohol-associated cues also in humans (Myrick et al., 2004; Filbey et al., 2008; Gilman et al., 2008; Vollstadt-Klein et al., 2020). Other brain regions comprising the basic motivational circuit emerging from human imaging include the orbitofrontal/medial aspects of the prefrontal cortex and insular cortex, both found to be activated also in $\mathrm{AA}$ and $\mathrm{msP}$ rats in the present study. The insula has been implicated in interoceptive effects of drug taking, and its contribution to drug-related responses has been demonstrated both in humans and animal models including the AA line (Contreras et al., 2007; Naqvi and Bechara, 2009; Droutman et al., 2015; Haaranen et al., 2020). Interestingly, ventral striatal and insula activation to alcohol cues was more amplified in high drinking than low drinking humans (Ihssen et al., 2011), and the projection from the insula to nucleus accumbens is involved in alcohol consumption both in rodents and human alcoholics (Seif et al., 2013; Grodin et al., 2018; Jaramillo et al., 2018). Collectively, the activation of the common brain regions in AA and msP rats, including the nucleus accumbens, medial prefrontal cortex and insula, is an expected effect of alcohol cues and the presence of alcohol in the body, but it may also reflect the motivational processes driving alcohol drinking in these animals.

Apart from these overlapping structures, the markedly different overall activation patterns in these lines could be related to their genetic background and the resulting behavioral and neurochemical differences. In the AA rats, the lack of effect by mesolimbic dopamine depletion on alcohol drinking suggests that alcohol reinforcement could be mediated also through other systems in these rats (Koistinen et al., 2001). The major activation cluster found in the bed nucleus of the stria terminalis (BNST) and various hypothalamic nuclei supports this view. The BNST has been strongly implicated 
in stress responses, but its nuclei are also closely connected with the key components of circuitry that mediates positive reinforcement, including VTA, ventral striatum, and hypothalamic nuclei (Wills and Winder, 2013). Various transmitter systems of the BNST, such as dopamine and GABA, have been associated with alcohol reinforcement (Hyytia and Koob, 1995; Carboni et al., 2000; Eiler et al., 2003). Also, more chronic alcohol exposures (Olive et al., 2002) or alcohol-associated environmental cues (Hill et al., 2007) altered BNST functioning, further demonstrating its importance for alcohol-motivated behavior. Since the BNST is bidirectionally connected with hypothalamic nuclei (Alheid and Heimer, 1988), the observed activation cluster suggests particular relevance for the BNST and hypothalamus for mediating alcohol reinforcement in AA rats. Various neuropeptides in hypothalamic regions regulate alcohol drinking in a manner dissociable from a generalized appetitive motivation control, including orexin-containing neurons localized in the dorsomedial, perifornical, and lateral hypothalamus (Peyron et al., 1998; Walker and Lawrence, 2017), as well as oxytocin-containing neurons in the medial hypothalamus (Peters et al., 2017; Hansson et al., 2018; Tunstall et al., 2019). Therefore, the hypothalamic activation cluster in AA rats could pinpoint an important, previously unrecognized region for mediating voluntary alcohol drinking in these rats.

In sharp contrast to AA rats displaying widespread subcortical and cortical activation, the pattern found in msP rats was highly restricted to the rostral and ventral parts of the striatum and medial prefrontal cortex. These differences are probably not due to the lower $\mathrm{Mn}^{2+}$ dose used in msP rats, because the average signal enhancement was slightly higher in msP rats and the differences in activation patterns were qualitative. The strong activation of the medial prefrontal cortex (mPFC) including the infralimbic and prelimbic areas seems therefore genuine in msP rats, and a property that this line shares with the post-dependent model, in which rodents are exposed to high blood alcohol levels during chronic intermittent ethanol (Meinhardt and Sommer, 2015). Prolonged alcohol exposure caused a profound reprogramming in the $\mathrm{MPFC}$, leading to molecular dysregulation (Heilig et al., 2017) and alterations in activity and connectivity (Scuppa et al., 2020) 
that probably contribute to the alcohol-dependent phenotype. Similar alterations appear to preexist in the msP line (Bjork et al., 2010; Bifone et al., 2019). Both the infralimbic and prelimbic cortices have been implicated in fear and drug-seeking, with a dorsal-ventral distinction. Thus, the prelimbic cortex has been suggested to drive the expression of fear and drug seeking, whereas the more ventral infralimbic cortex promotes the extinction of conditioned fear and drug seeking (Peters et al., 2009). Given these seemingly competing roles of the dorsal and ventral medial prefrontal cortex in fear and drug seeking, the functional significance of the observed activation in both areas is not clear. However, it could be hypothesized that activation of the infralimbic prefrontal cortex by alcohol drinking in $\mathrm{msP}$ rats may be related to inhibition of the innate anxiety-like behavior in this line (Ciccocioppo et al., 2006; Pfarr et al., 2015), whereas the prelimbic cortex activation mediates alcohol-seeking behavior. The infralimbic cortex activation inhibits the central amygdala (Quirk et al., 2003), which is in line with a previous finding that alcohol drinking decreased the anxietyassociated overactivity of corticotropin-releasing hormone receptor 1 (CRH-R1) in the amygdala in msP rats (Hansson et al., 2006; Hansson et al., 2007). In the present experiments, however, no evidence for decreased activity either in $\mathrm{msP}$ or AA rats was found. This may reflect the limited ability of MEMRI to detect deactivation, as the enhanced $\mathrm{T} 1$ signals are produced by $\mathrm{Mn}^{2+}$ entry associated with neuronal activation. In further support for the involvement of the infralimbic cortex in alcohol effects, chronic intermittent alcohol exposure attenuated mGluR2/3 autoreceptor function and NMDA-receptor mediated currents in the pyramidal neurons of the infralimbic cortex, which was related to persistent escalation of alcohol self-administration and deficient fear extinction (Holmes et al., 2012; Meinhardt et al., 2013).

Laboratory-specific differences in hardware and imaging parameters could contribute to either the quality of the data or the patterns of brain activation and can therefore be considered a limitation of this study. However, contrast enhancement in the $\mathrm{T}_{1}$-weigted MR images produced by $\mathrm{Mn}^{2+}$ accumulation is very robust, and it is not likely that minor differences in sequence parameters could have contributed to the differential activation pattern in these rat strain. In addition, we saw more 
widespread brain activation in the AA rats with a lower 4.7T field than the restricted frontal pattern in the msP rats imaged using a higher 7T field strength and a higher SNR. In both cases, the spatial localization of activation was detailed and region-specific, suggesting that we captured qualitatively different brain activation patterns in these two rat strains.

Taken together, the patterns of functional brain activation induced by voluntary alcohol drinking in AA and msP rats appear to recapitulate the pivotal elements of the motivational circuits underlying alcohol and drug taking suggested by previous animal and human studies. However, the intriguing novel finding was that despite similar alcohol consumption levels, the neural circuits activated by alcohol drinking were conspicuously different in these rat lines. One way of interpreting these differences is to relate them to the co-segregated behavioral traits, i.e., risk-taking and impulsivity in the AA and anxiety-like behavior in the msP rats, and to claim that the observed dissimilarity in functional circuits reflects innately different behavioral processes and states driving alcohol drinking. The current data may also suggest potential neuroanatomical substrates for the heterogeneity seen in human alcoholic patients. The brain networks driving alcohol drinking in the animal models could be subjected for further circuit-level interrogation for pharmacological treatment effects (Dudek et al., 2016). Comparison of those data with respective human imaging outcomes could facilitate our understanding of dimensions of behavior and identify targets for treatment (Heilig et al., 2016; De Santis et al., 2019; Heilig et al., 2019; De Santis et al., 2020). Such translational framework for AUD is needed to fully implement recent proposals to transcend categorical psychiatric diagnosis such as the Research Domain Criteria (RDoC) and Addictions Neuroclinical Assessment initiatives (Kwako et al., 2017). 


\section{REFERENCES}

Alheid GF, Heimer L (1988) New perspectives in basal forebrain organization of special relevance for neuropsychiatric disorders: the striatopallidal, amygdaloid, and corticopetal components of substantia innominata. Neuroscience 27:1-39.

Bach P, Weil G, Pompili E, Hoffmann S, Hermann D, Vollstadt-Klein S, Mann K, Perez-Ramirez U, Moratal D, Canals S, Dursun SM, Greenshaw AJ, Kirsch P, Kiefer F, Sommer WH (2020) Incubation of neural alcohol cue reactivity after withdrawal and its blockade by naltrexone. Addict Biol 25:e12717.

Bachtell RK, Wang YM, Freeman P, Risinger FO, Ryabinin AE (1999) Alcohol drinking produces brain region-selective changes in expression of inducible transcription factors. Brain Res 847:157-165.

Bangasser DA, Lee CS, Cook PA, Gee JC, Bhatnagar S, Valentino RJ (2013) Manganese-enhanced magnetic resonance imaging (MEMRI) reveals brain circuitry involved in responding to an acute novel stress in rats with a history of repeated social stress. Physiol Behav 122:228236.

Bifone A, Gozzi A, Cippitelli A, Matzeu A, Domi E, Li H, Scuppa G, Cannella N, Ubaldi M, Weiss F, Ciccocioppo R (2019) phMRI, neurochemical and behavioral responses to psychostimulants distinguishing genetically selected alcohol-preferring from genetically heterogenous rats. Addict Biol 24:981-993.

Bjork K, Hansson AC, Sommer WH (2010) Genetic variation and brain gene expression in rodent models of alcoholism implications for medication development. Int Rev Neurobiol 91:129171.

Carboni E, Silvagni A, Rolando MT, Di Chiara G (2000) Stimulation of in vivo dopamine transmission in the bed nucleus of stria terminalis by reinforcing drugs. J Neurosci 20:RC102. 
Ciccocioppo R (2013) Genetically selected alcohol preferring rats to model human alcoholism. Curr Top Behav Neurosci 13:251-269.

Ciccocioppo R, Economidou D, Cippitelli A, Cucculelli M, Ubaldi M, Soverchia L, Lourdusamy A, Massi M (2006) Genetically selected Marchigian Sardinian alcohol-preferring (msP) rats: an animal model to study the neurobiology of alcoholism. Addict Biol 11:339-355.

Colombo G, Agabio R, Lobina C, Reali R, Vacca G, Gessa GL (1998) Stimulation of locomotor activity by voluntarily consumed ethanol in Sardinian alcohol-preferring rats. Eur J Pharmacol 357:109-113.

Colombo G, Agabio R, Lobina C, Reali R, Zocchi A, Fadda F, Gessa GL (1995) Sardinian alcoholpreferring rats: a genetic animal model of anxiety. Physiol Behav 57:1181-1185.

Contreras M, Ceric F, Torrealba F (2007) Inactivation of the interoceptive insula disrupts drug craving and malaise induced by lithium. Science 318:655-658.

Courtney KE, Ghahremani DG, Ray LA (2016) The Effects of Pharmacological Opioid Blockade on Neural Measures of Drug Cue-Reactivity in Humans. Neuropsychopharmacology 41:28722881.

De Montis MG, Grappi S, Gambarana C, Leggio B, Nanni G, Scheggi S, Tagliamonte A (2004) Sardinian alcohol-preferring rats show low 5-HT extraneuronal levels in the MPFC and no habituation in monoaminergic response to repeated ethanol consumption in the NAcS. Brain Res 1006:18-27.

De Santis S, Bach P, Perez-Cervera L, Cosa-Linan A, Weil G, Vollstadt-Klein S, Hermann D, Kiefer F, Kirsch P, Ciccocioppo R, Sommer WH, Canals S (2019) Microstructural White Matter Alterations in Men With Alcohol Use Disorder and Rats With Excessive Alcohol Consumption During Early Abstinence. JAMA Psychiatry 76:749-758.

De Santis S, Cosa-Linan A, Garcia-Hernandez R, Dmytrenko L, Vargova L, Vorisek I, Stopponi S, Bach P, Kirsch P, Kiefer F, Ciccocioppo R, Sykova E, Moratal D, Sommer WH, Canals S (2020) 
Chronic alcohol consumption alters extracellular space geometry and transmitter diffusion in the brain. Science Advances. Accepted.

Droutman V, Read SJ, Bechara A (2015) Revisiting the role of the insula in addiction. Trends Cogn Sci 19:414-420.

Dudek M, Canals S, Sommer WH, Hyytia P (2016) Modulation of nucleus accumbens connectivity by alcohol drinking and naltrexone in alcohol-preferring rats: A manganese-enhanced magnetic resonance imaging study. Eur Neuropsychopharmacol 26:445-455.

Dudek M, Abo-Ramadan U, Hermann D, Brown M, Canals S, Sommer WH, Hyytia P (2015) Brain activation induced by voluntary alcohol and saccharin drinking in rats assessed with manganese-enhanced magnetic resonance imaging. Addict Biol 20:1012-1021.

Eiler WJ, 2nd, Seyoum R, Foster KL, Mailey C, June HL (2003) D1 dopamine receptor regulates alcohol-motivated behaviors in the bed nucleus of the stria terminalis in alcohol-preferring (P) rats. Synapse 48:45-56.

Eriksson K (1968) Genetic selection for voluntary alcohol consumption in the albino rat. Science 159:739-741.

Eschenko O, Canals S, Simanova I, Logothetis NK (2010a) Behavioral, electrophysiological and histopathological consequences of systemic manganese administration in MEMRI. Magn Reson Imaging 28:1165-1174.

Eschenko O, Canals S, Simanova I, Beyerlein M, Murayama Y, Logothetis NK (2010b) Mapping of functional brain activity in freely behaving rats during voluntary running using manganeseenhanced MRI: implication for longitudinal studies. Neuroimage 49:2544-2555.

Filbey FM, Claus E, Audette AR, Niculescu M, Banich MT, Tanabe J, Du YP, Hutchison KE (2008) Exposure to the taste of alcohol elicits activation of the mesocorticolimbic neurocircuitry. Neuropsychopharmacology 33:1391-1401. 
Gildish I, Manor D, David O, Sharma V, Williams D, Agarwala U, Wang X, Kenney JW, Proud CG, Rosenblum K (2012) Impaired associative taste learning and abnormal brain activation in kinase-defective eEF2K mice. Learn Mem 19:116-125.

Gilman JM, Ramchandani VA, Crouss T, Hommer DW (2012a) Subjective and neural responses to intravenous alcohol in young adults with light and heavy drinking patterns. Neuropsychopharmacology 37:467-477.

Gilman JM, Ramchandani VA, Davis MB, Bjork JM, Hommer DW (2008) Why we like to drink: a functional magnetic resonance imaging study of the rewarding and anxiolytic effects of alcohol. J Neurosci 28:4583-4591.

Gilman JM, Smith AR, Ramchandani VA, Momenan R, Hommer DW (2012b) The effect of intravenous alcohol on the neural correlates of risky decision making in healthy social drinkers. Addict Biol 17:465-478.

Grodin EN, Ray LA (2019) The Use of Functional Magnetic Resonance Imaging to Test Pharmacotherapies for Alcohol Use Disorder: A Systematic Review. Alcohol Clin Exp Res 43:2038-2056.

Grodin EN, Sussman L, Sundby K, Brennan GM, Diazgranados N, Heilig M, Momenan R (2018) Neural Correlates of Compulsive Alcohol Seeking in Heavy Drinkers. Biological psychiatry Cognitive neuroscience and neuroimaging 3:1022-1031.

Haaranen M, Scuppa G, Tambalo S, Järvi V, Bertozzi S, Armirotti A, Sommer WH, Bifone A, Hyytiä P (2020) Anterior Insula Stimulation Suppresses Appetitive Behavior While Inducing Forebrain Activation in Alcohol-Preferring Rats. Translational psychiatry. 2020 May 18;10(1):150.

Hansson AC, Cippitelli A, Sommer WH, Ciccocioppo R, Heilig M (2007) Region-specific downregulation of Crhr1 gene expression in alcohol-preferring msP rats following ad lib access to alcohol. Addict Biol 12:30-34.

Hansson AC, Rimondini R, Neznanova O, Sommer WH, Heilig M (2008) Neuroplasticity in brain reward circuitry following a history of ethanol dependence. Eur J Neurosci 27:1912-1922. 
Hansson AC, Cippitelli A, Sommer WH, Fedeli A, Bjork K, Soverchia L, Terasmaa A, Massi M, Heilig M, Ciccocioppo R (2006) Variation at the rat Crhr1 locus and sensitivity to relapse into alcohol seeking induced by environmental stress. Proc Natl Acad Sci U S A 103:1523615241.

Hansson AC, Koopmann A, Uhrig S, Buhler S, Domi E, Kiessling E, Ciccocioppo R, Froemke RC, Grinevich V, Kiefer F, Sommer WH, Vollstadt-Klein S, Spanagel R (2018) Oxytocin Reduces Alcohol Cue-Reactivity in Alcohol-Dependent Rats and Humans. Neuropsychopharmacology 43:1235-1246.

Heilig M, Sommer WH, Spanagel R (2016) The Need for Treatment Responsive Translational Biomarkers in Alcoholism Research. Curr Top Behav Neurosci 28:151-171.

Heilig M, Goldman D, Berrettini W, O'Brien CP (2011) Pharmacogenetic approaches to the treatment of alcohol addiction. Nat Rev Neurosci 12:670-684.

Heilig M, Augier E, Pfarr S, Sommer WH (2019) Developing neuroscience-based treatments for alcohol addiction: A matter of choice? Translational psychiatry 9:255.

Heilig M, Barbier E, Johnstone AL, Tapocik J, Meinhardt MW, Pfarr S, Wahlestedt C, Sommer WH (2017) Reprogramming of mPFC transcriptome and function in alcohol dependence. Genes Brain Behav 16:86-100.

Heinz A et al. (2020) Addiction Research Consortium: Losing and regaining control over drug intake (ReCoDe)-From trajectories to mechanisms and interventions. Addict Biol 25:e12866.

Hill KG, Ryabinin AE, Cunningham CL (2007) FOS expression induced by an ethanol-paired conditioned stimulus. Pharmacol Biochem Behav 87:208-221.

Holmes A, Fitzgerald PJ, MacPherson KP, DeBrouse L, Colacicco G, Flynn SM, Masneuf S, Pleil KE, Li C, Marcinkiewcz CA, Kash TL, Gunduz-Cinar O, Camp M (2012) Chronic alcohol remodels prefrontal neurons and disrupts NMDAR-mediated fear extinction encoding. Nat Neurosci 15:1359-1361. 
Hyytia P, Koob GF (1995) GABAA receptor antagonism in the extended amygdala decreases ethanol self-administration in rats. Eur J Pharmacol 283:151-159.

Ihssen N, Cox WM, Wiggett A, Fadardi JS, Linden DE (2011) Differentiating heavy from light drinkers by neural responses to visual alcohol cues and other motivational stimuli. Cereb Cortex 21:1408-1415.

Jaramillo AA, Randall PA, Stewart S, Fortino B, Van Voorhies K, Besheer J (2018) Functional role for cortical-striatal circuitry in modulating alcohol self-administration. Neuropharmacology 130:42-53.

Jenkinson M, Bannister P, Brady M, Smith S (2002) Improved optimization for the robust and accurate linear registration and motion correction of brain images. Neuroimage 17:825841.

Koistinen M, Tuomainen P, Hyytia P, Kiianmaa K (2001) Naltrexone suppresses ethanol intake in 6hydroxydopamine-treated rats. Alcohol Clin Exp Res 25:1605-1612.

Koob GF, Roberts AJ, Schulteis G, Parsons LH, Heyser CJ, Hyytia P, Merlo-Pich E, Weiss F (1998) Neurocircuitry targets in ethanol reward and dependence. Alcohol Clin Exp Res 22:3-9. Kwako LE, Momenan R, Grodin EN, Litten RZ, Koob GF, Goldman D (2017) Addictions Neuroclinical Assessment: A reverse translational approach. Neuropharmacology 122:254-264.

Laine MA, Sokolowska E, Dudek M, Callan SA, Hyytia P, Hovatta I (2017) Brain activation induced by chronic psychosocial stress in mice. Scientific reports 7:15061.

Mardones J, Segovia N, Hederra A (1953) Heredity of experimental alcohol preference in rats. II. Coefficient of heredity. Q J Stud Alcohol 14:1-2.

McBride WJ, Li TK (1998) Animal models of alcoholism: neurobiology of high alcohol-drinking behavior in rodents. Crit Rev Neurobiol 12:339-369.

McGuire JL, Bergstrom HC, Parker CC, Le T, Morgan M, Tang H, Selwyn RG, Silva AC, Choi K, Ursano RJ, Palmer AA, Johnson LR (2013) Traits of fear resistance and susceptibility in an advanced intercross line. Eur J Neurosci 38:3314-3324. 
Meinhardt MW, Sommer WH (2015) Postdependent state in rats as a model for medication development in alcoholism. Addict Biol 20:1-21.

Meinhardt MW, Hansson AC, Perreau-Lenz S, Bauder-Wenz C, Stahlin O, Heilig M, Harper C, Drescher KU, Spanagel R, Sommer WH (2013) Rescue of infralimbic mGluR2 deficit restores control over drug-seeking behavior in alcohol dependence. J Neurosci 33:27942806.

Moller C, Wiklund L, Thorsell A, Hyytia P, Heilig M (1997) Decreased measures of experimental anxiety in rats bred for high alcohol preference. Alcohol Clin Exp Res 21:656-660.

Myrick H, Anton RF, Li X, Henderson S, Drobes D, Voronin K, George MS (2004) Differential brain activity in alcoholics and social drinkers to alcohol cues: relationship to craving. Neuropsychopharmacology 29:393-402.

Naqvi NH, Bechara A (2009) The hidden island of addiction: the insula. Trends Neurosci 32:56-67. Noori HR, Spanagel R, Hansson AC (2012) Neurocircuitry for modeling drug effects. Addict Biol 17:827-864.

Nurmi M, Sinclair JD, Kiianmaa K (1998) Dopamine release during ethanol drinking in AA rats. Alcohol ClinExpRes 22:1628-1633.

Olive MF, Koenig HN, Nannini MA, Hodge CW (2002) Elevated extracellular CRF levels in the bed nucleus of the stria terminalis during ethanol withdrawal and reduction by subsequent ethanol intake. Pharmacol Biochem Behav 72:213-220.

Paivarinta P, Korpi ER (1993) Voluntary ethanol drinking increases locomotor activity in alcoholpreferring AA rats. Pharmacol Biochem Behav 44:127-132.

Paxinos G, Watson C (2007) The Rat Brain in Stereotaxic Coordinates, 6th ed. Academic Press, London.

Peters J, Kalivas PW, Quirk GJ (2009) Extinction circuits for fear and addiction overlap in prefrontal cortex. Learn Mem 16:279-288. 
Peters ST, Bowen MT, Bohrer K, McGregor IS, Neumann ID (2017) Oxytocin inhibits ethanol consumption and ethanol-induced dopamine release in the nucleus accumbens. Addict Biol 22:702-711.

Peyron C, Tighe DK, van den Pol AN, de Lecea L, Heller HC, Sutcliffe JG, Kilduff TS (1998) Neurons containing hypocretin (orexin) project to multiple neuronal systems. J Neurosci 18:999610015.

Pfarr S, Meinhardt MW, Klee ML, Hansson AC, Vengeliene V, Schonig K, Bartsch D, Hope BT, Spanagel R, Sommer WH (2015) Losing Control: Excessive Alcohol Seeking after Selective Inactivation of Cue-Responsive Neurons in the Infralimbic Cortex. J Neurosci 35:1075010761.

Porrino LJ, WilliamsHemby L, Whitlow C, Bowen C, Samson HH (1998) Metabolic mapping of the effects of oral alcohol self-administration in rats. Alcohol ClinExpRes 22:176-182.

Quirk GJ, Likhtik E, Pelletier JG, Pare D (2003) Stimulation of medial prefrontal cortex decreases the responsiveness of central amygdala output neurons. J Neurosci 23:8800-8807.

Roman E, Meyerson BJ, Hyytia P, Nylander I (2007) The multivariate concentric square field test reveals different behavioural profiles in male AA and ANA rats with regard to risk taking and environmental reactivity. Behav Brain Res 183:195-205.

Schacht JP, Randall PK, Latham PK, Voronin KE, Book SW, Myrick H, Anton RF (2017) Predictors of Naltrexone Response in a Randomized Trial: Reward-Related Brain Activation, OPRM1 Genotype, and Smoking Status. Neuropsychopharmacology 42:2640-2653.

Schwarz AJ, Danckaert A, Reese T, Gozzi A, Paxinos G, Watson C, Merlo-Pich EV, Bifone A (2006) A stereotaxic MRI template set for the rat brain with tissue class distribution maps and coregistered anatomical atlas: application to pharmacological MRI. Neuroimage 32:538-550.

Scuppa G, Tambalo S, Pfarr S, Sommer WH, Bifone A (2020) Aberrant insular cortex connectivity in abstinent alcohol-dependent rats is reversed by dopamine D3 receptor blockade. Addict Biol 25:e12744. 
Seif T, Chang SJ, Simms JA, Gibb SL, Dadgar J, Chen BT, Harvey BK, Ron D, Messing RO, Bonci A, Hopf FW (2013) Cortical activation of accumbens hyperpolarization-active NMDARs mediates aversion-resistant alcohol intake. Nat Neurosci 16:1094-1100.

Silva AC, Lee JH, Aoki I, Koretsky AP (2004) Manganese-enhanced magnetic resonance imaging (MEMRI): methodological and practical considerations. NMR Biomed 17:532-543.

Smith SM (2002) Fast robust automated brain extraction. Hum Brain Mapp 17:143-155.

Sommer W, Hyytia P, Kiianmaa K (2006) The alcohol-preferring AA and alcohol-avoiding ANA rats: neurobiology of the regulation of alcohol drinking. Addict Biol 11:289-309.

Spanagel R (2009) Alcoholism: A Systems Approach From Molecular Physiology to Addictive Behavior. Physiol Rev 89:649-705.

Tunstall BJ, Kirson D, Zallar L, McConnell SA, Vendruscolo JCM, Ho CP, Oleata CS, Khom S, Manning M, Lee MR, Leggio L, Koob GF, Roberto M, Vendruscolo LF (2019) Oxytocin blocks enhanced motivation for alcohol in alcohol dependence and blocks alcohol effects on GABAergic transmission in the central amygdala. PLoS Biol 17:e2006421.

Walker LC, Lawrence AJ (2017) The Role of Orexins/Hypocretins in Alcohol Use and Abuse. Curr Top Behav Neurosci 33:221-246.

Wills TA, Winder DG (2013) Ethanol effects on N-methyl-D-aspartate receptors in the bed nucleus of the stria terminalis. Cold Spring Harb Perspect Med 3:a012161.

Vilpoux C, Warnault V, Pierrefiche O, Daoust M, Naassila M (2009) Ethanol-sensitive brain regions in rat and mouse: a cartographic review, using immediate early gene expression. Alcohol Clin Exp Res 33:945-969.

Vollstadt-Klein S, Wichert S, Rabinstein J, Buhler M, Klein O, Ende G, Hermann D, Mann K (2010) Initial, habitual and compulsive alcohol use is characterized by a shift of cue processing from ventral to dorsal striatum. Addiction 105:1741-1749.

Vollstadt-Klein S, Gerhardt S, Lee A, Strosche A, Sharafi G, Nuriyeva R, Seidt J, Hennig O, Alm B, Hermann D, Sommer WH, Kiefer F, Luderer M, Sobanski E (2020) Interaction between 
behavioral inhibition and neural alcohol cue-reactivity in ADHD and alcohol use disorder.

Psychopharmacology (Berl).

Yang J, Li Q (2020) Manganese-Enhanced Magnetic Resonance Imaging: Application in Central Nervous System Diseases. Front Neurol 11:143. 


\section{FIGURE LEGENDS}

Figure 1. Effects of the seven-day $\mathrm{MnCl}_{2}$ infusion with osmotic minipumps on alcohol consumption in $\mathrm{msP}(n=14)$ and AA rats $(n=10)$. Data present mean $( \pm S E M)$ in each group. ${ }^{*} P<0.05$, significantly different from the baseline (BL), defined as the mean of the previous seven days (Dunnett's test).

Figure 2. Brain activity maps during alcohol drinking in AA rats. $T_{1}$-weighted MRI signal enhancement was found in alcohol drinking rats compared to water-drinking control animals. Only signal enhancements were found (alcohol > water). Shown are coronal (A), sagittal (B) and horizontal (C) functional MEMRI maps threshold at $p<0.05$ (two-tailed t-test, corrected, $n=10 /$ group) and overlaid on representative $T_{1}$-weighted images. Color code represents $t$-values. Numbers in the leftbottom corner of each image indicate the positions of the sections from bregma in millimeters. Abbreviations: Acb, nucleus accumbens; Ins, Insular cortex (mainly agranular); $\mathrm{Cg}$, cingulate cortex; BNST, bed nucleus of the stria terminalis; vHC, ventral hippocampus; LH, lateral hypothalamus; LS, lateral septum; Pir, piriform cortex; $\mathrm{CeA}$, central amygdaloid nucleus; $\mathrm{AH}$, anterior hypothalamus.

Figure 3. Brain activity maps during alcohol drinking in msP rats. $\mathrm{T}_{1}$-weighted $\mathrm{MRI}$ signal enhancement was found in alcohol drinking rats compared to water-drinking control animals. Only signal enhancements were found (alcohol > water). Shown are coronal (left colum) and horizontal (right column) MEMRI maps obtained using osmotic minipumps for manganese infusion $(p<0.05$, two-tailed t-test, corrected, $n=14 /$ group). Color code represents t-values. Numbers in the rightbottom corner of each image indicate the positions of the sections from bregma in millimeters. Abbreviations: PrL, prelimbic cortex; IL, infralimbic cortex; AON, anterior olfactory nucleus; Ins, Insular cortex (mainly agranular); $\mathrm{Acb}$, nucleus accumbens; $\mathrm{Cg}$, cingulate cortex; $\mathrm{CPu}$, caudate putamen.

Figure 4. Distinct brain networks are activated by alcohol drinking in $A A$ and $m s P$ rats. Brain regions with enhanced manganese accumulation in AA (red) and msP (blue) rats are co-registered and overlaid on corresponding sagittal (A) and coronal (B-C) slices of the Paxinos and Watson (2007) rat 
brain atlas. Statistical thresholds are the same used in Figs 2 and 3. Dotted lines in (A) mark the position of the coronal slices in (B) and (C), and vice versa. The combined representation of $A A$ and msP MEMRI maps for the complete brain volume can be seen in Movie 1. (D) Histogram representation of the volume of specific brain regions activated by alcohol drinking (in \% of the atlasdefined total volume of each region) in $\mathrm{AA}$ (red) and msP (blue) rats. The list of brain regions was composed both based on size of the activated areas in msP and AA rats and the proposed neurocircuitry of alcohol effects (Noori et al., 2012). Abbreviations: OB, olfactory bulb; AON, anterior olfactory nucleus; PirC, piriform cortex; EndP, endopiriform cortex; OC, orbitofrontal cortex; FrA, frontal association cortex; PrL, prelimbic cortex; IL, infralimbic cortex; Ins, insula; $\mathrm{Cg}$, cingulate cortex; M1, primary motor cortex; M2, secondary motor cortex; S1, primary somatosensory cortex; S2, secondary somatosensory cortex; HC, hippocampus; PRh, perirhinal cortex; EctC, ectorhinal cortex; TeA, temporal association cortex; Acb, nucleus accumbens; CPu, caudate putamen; VP, ventral pallidum; Amy, amygdala; Bed; bed nucleus of stria terminalis; PO, preoptic area; $\mathrm{H}$, hypothalamus; SN, substantia nigra; PAG, periaqueductal grey.

Movie 1. Combined representation of AA and msP MEMRI maps ( $p<0.05$, corrected) across the complete brain volume. Brain regions with enhanced manganese accumulation in AA (red) and msP (blue) rats are co-registered and overlaid on an anatomical image. Each frame of the movie represents one coronal slice. 


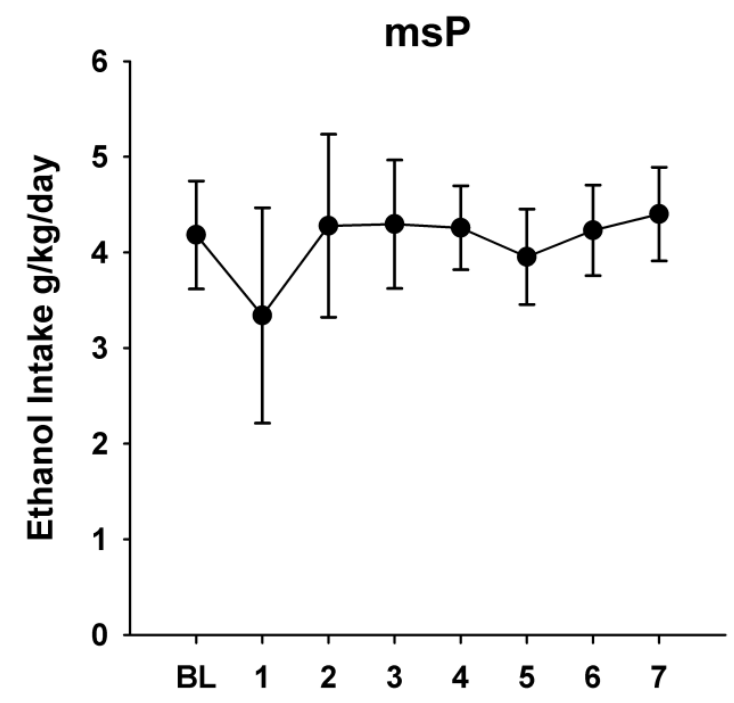

Manganese Infusion Days

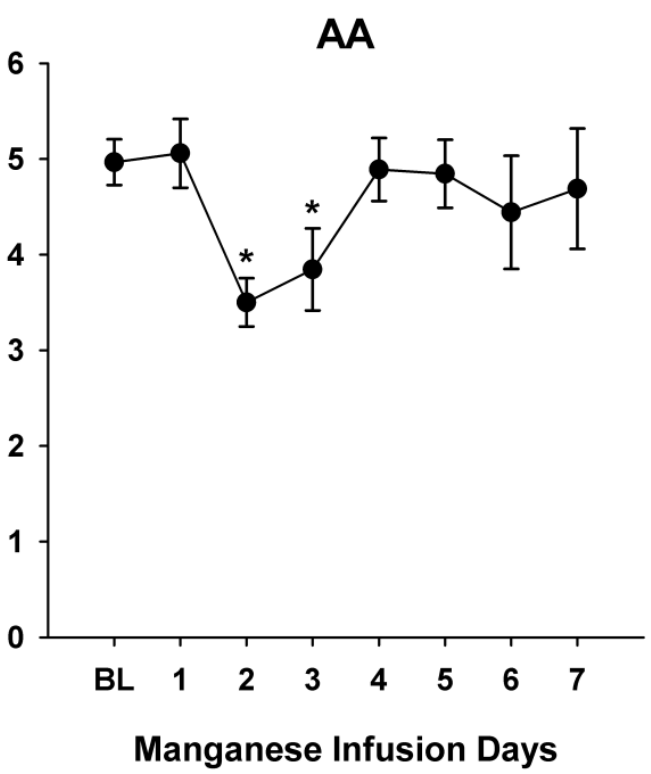

Manganese Infusion Days

Figure 1

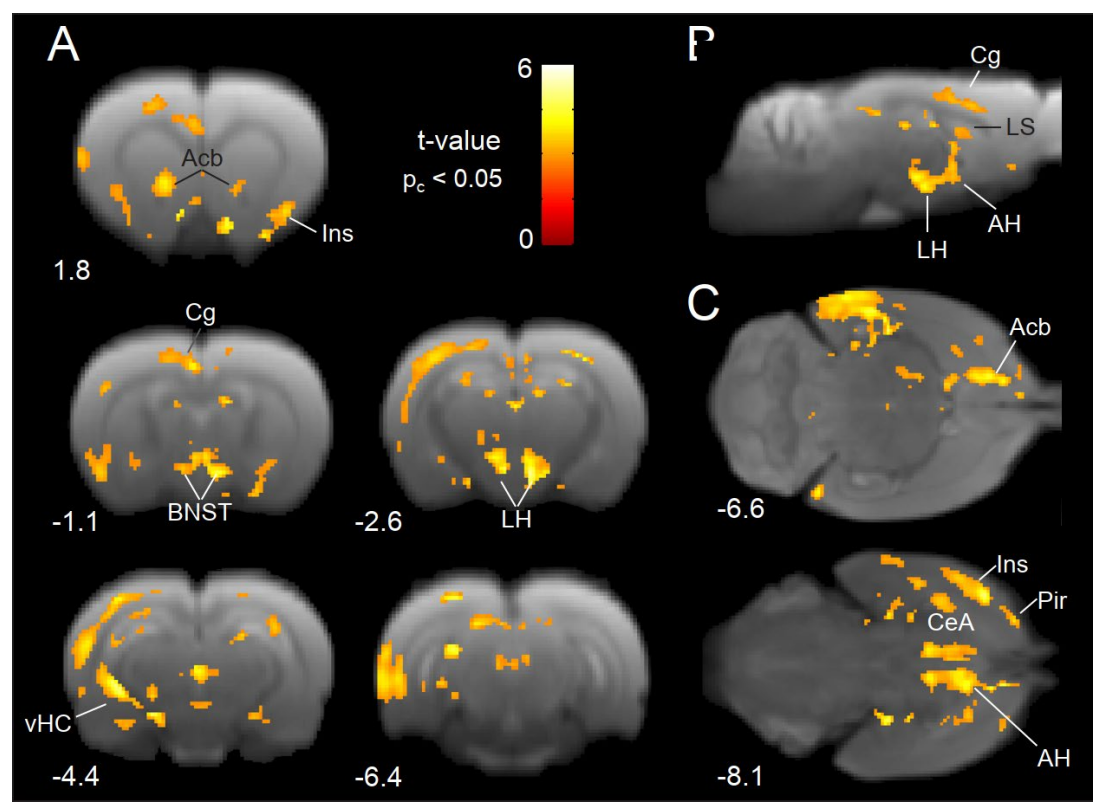

Figure 2 


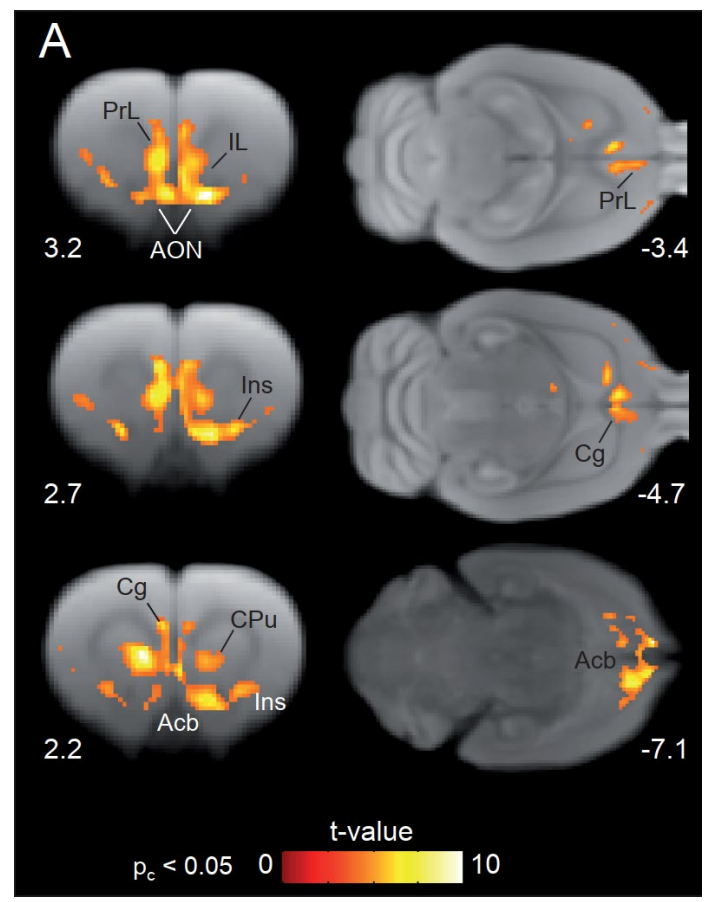

Figure 3.

A

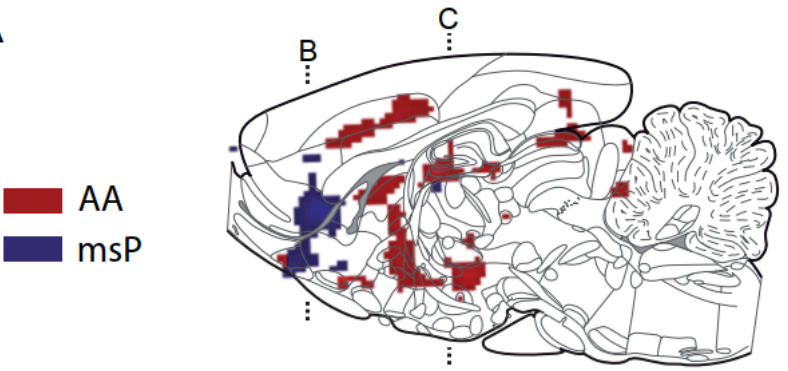

B

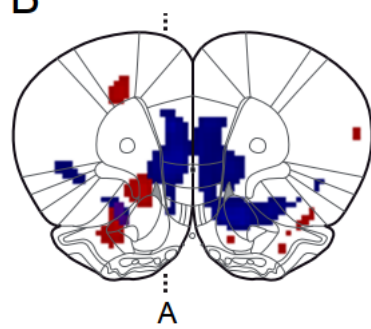

$\mathrm{C}$

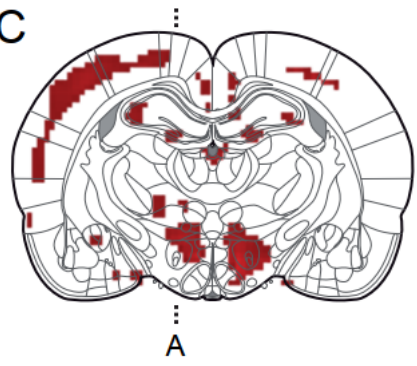

D $\%$ of region activation

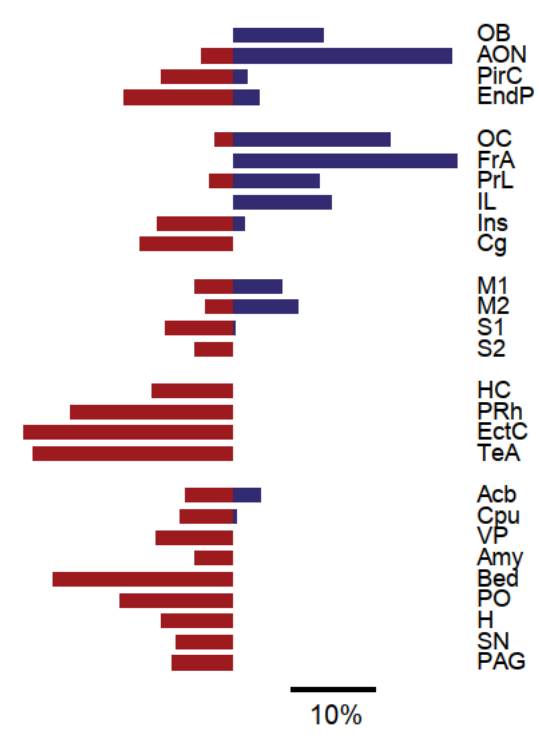

Figure 4. 